\title{
THE EXISTENCE OF PERIODIC SOLUTIONS FOR A CLASS OF NEUTRAL DIFFERENTIAL DIFFERENCE EQUATIONS
}

\author{
YONGSHAO CHEN ${ }^{1}$
}

(Received 15 May 1990; revised 14 May 1991)

\begin{abstract}
In this paper, we study the existence of periodic solutions of the NDDE (neutral differential difference equation):

$$
(x(t)+c x(t-\tau))^{\prime}=-f(x(t), x(t-\tau))
$$

where $\tau>0$ and $c$ is a real number. We obtain a sufficient condition under which (*) has at least $k$ nonconstant oscillatory periodic solutions.
\end{abstract}

\section{Introduction}

In 1967, R. Brayton [1-2] considered the problem of lossless transmission lines used to connect switching circuits and obtained the following NDDE (neutral differential difference equation):

$$
\dot{u}(t)-k \dot{u}(t-2 / s)=f(u(t), u(t-2 / s))
$$

where $s=\sqrt{L C}$. In this paper, we study the existence of a periodic solution of (A). For the case $k=0$, several papers [5-6] have given sufficient conditions for the existence of a periodic solution of (A). However, for the cases $k \neq 0$, there are few papers dealing with the existence of a periodic solution of (A). Now, we consider a class of NDDEs which is more general than (A):

$$
(x(t)+c x(t-\tau))^{\prime}=-f(x(t), x(t-\tau))
$$

where $\tau>0, c$ is a real number, and $f(x, y)$ is a continuous function. Since the solutions of (1) may not be differentiable, (1) is more general than (A).

\footnotetext{
${ }^{1}$ Department of Mathematics, South China Normal University, Guangzhou, 510631, China.

(C) Copyright Australian Mathematical Society 1992, Serial-fee code 0334-2700/92
} 
Throughout this paper we assume that there exists a continuous function $g(x, y)$ such that

$$
f(x, y)=g(x, y)-c g(y, x) .
$$

Usually a function $g(x, y)$ which satisfies (2) is easily obtained. For example if $c \neq \pm 1$, then, from (2), we have

$$
f(y, x)=g(y, x)-c g(x, y) .
$$

By (2), (3) we obtain

$$
g(x, y)=\frac{1}{1-c^{2}} f(x, y)+\frac{c}{1-c^{2}} f(y, x) .
$$

\section{The main result}

Consider the ordinary differential system:

$$
\frac{d x}{d y}=-g(x, y), \quad \frac{d y}{d x}=g(y, x) .
$$

We suppose that

(I) $g(x, y)$ is continuous on $R^{2}$;

(II) $\left(\begin{array}{c}-g(x, y) \\ g(y, x)\end{array}\right)$ satisfies the local Lipschitz condition on $R^{2}$.

It is easy to see that, under the conditions (I) and (II), (4) has a unique solution which satisfies the initial conditions $x\left(t_{0}\right)=x_{0}, y\left(t_{0}\right)=y_{0}$ and through any point $\left(x_{0}, y_{0}\right)$ (4) has a unique orbit [3].

\section{LEMMA. Suppose that}

(a) $g(x,-y)=-g(x, y), g(-x, y)=g(x, y), y g(x, y)>0 \quad(y \neq 0)$;

(b) there exists some $b>0$ such that

$$
g(y, x) / g(x, y) \leq A(x)^{\prime} B(y) \quad(x \geq 0, y \geq b>0),
$$

where $A(x)$ is continuous on $[0,+\infty), B(y)$ is continuous on $[b,+\infty)$, $B(y)>0 \quad(y \geq b)$ and $\int^{+\infty}(1 / B(y)) d y=+\infty$;

(c)

$$
\begin{aligned}
& \lim _{x^{2}+y^{2} \rightarrow 0^{+}} \frac{x g(y, x)+y g(x, y)}{x^{2}+y^{2}}=p, \\
& \lim _{x^{2}+y^{2} \rightarrow+\infty} \frac{x g(y, x)+y g(x, y)}{x^{2}+y^{2}}=q ;
\end{aligned}
$$

(d) there is some $T>0$ such that $p<2 \pi / T<q \leq+\infty$ or $q<2 \pi / T<$ $p \leq+\infty$. Then (4) has a periodic solution with period of $T$. 
Proof. Since $y g(x, y)>0(y \neq 0),(0,0)$ is the unique singular point of (4). By (I) and (II), through any point $\left(x_{0}, y_{0}\right)$, (4) has a unique orbit [6]. Assume that, through the point $\left(x_{0}, y_{0}\right)$, the orbit of (4) is $L$, where $x_{0} \geq 0$, $y_{0} \geq 0,\left(x_{0}, y_{0}\right) \neq(0,0)$. If $x_{0}=0$, then it is easy to see that $L$ intersects the positive $y$-axis. If $x_{0}>0$, then we claim that $L$ intersects the positive $y$-axis. Otherwise, by $d y / d x=-g(y, x) / g(x, y)<0 \quad(x>0, y>0), L$ has an asymptotic line $x=a \geq 0$. Let $L$ be $y=y(x) \quad\left(a<x \leq x_{0}\right)$. Then, by $\lim _{x \rightarrow a^{+}} y(x)=+\infty$, there is $x_{1}: a<x_{1} \leq x_{0}$ such that $y\left(x_{1}\right) \geq b$. Noting that $y(x)$ is decreasing, we have $y(x) \geq y\left(x_{1}\right) \geq b \quad\left(a<x \leq x_{1}\right)$. Hence, by the condition (b), we obtain

$$
\frac{d y(x)}{d x}=-\frac{g(y(x), x)}{g(x, y(x))} \geq-A(x) B(y(x))
$$

and

$$
\int_{x}^{x_{1}} \frac{d y(s)}{B(y(s))} \geq-\int_{x}^{x_{1}} A(s) d s
$$

or

$$
-\int_{y\left(x_{1}\right)}^{y(x)} \frac{d y}{B(y)} \geq-\int_{x}^{x_{1}} A(s) d s \quad\left(a<x \leq x_{1}\right) .
$$

As $x \rightarrow a^{+}$, the above inequality and the condition $\int^{+\infty}(1 / B(y)) d y=+\infty$ produce the desired contradiction and establish the claim that $L$ intersects the positive $y$-axis. By the condition (b) and (4), for $y \geq 0$ and $x \geq b>0$, we have

$$
\frac{d x}{d y}=-\frac{g(x, y)}{g(y, x)} \geq-A(y) B(x) .
$$

Similarly, we can prove that $L$ intersects the positive $x$-axis. Then, any orbit which passes through the point $\left(x_{0}, y_{0}\right)$ intersects the positive $x$-axis and the positive $y$-axis, where $x_{0} \geq 0, y_{0} \geq 0,\left(x_{0}, y_{0}\right) \neq(0,0)$. By (4) and the condition (a), we have $d y / d x=-g(y, x) / g(x, y), d x / d y=$ $-g(x, y) / g(y, x)$ and $g(x,-y)=-g(x, y), g(-x, y)=g(x, y)$. Hence, the orbit of (4) is symmetric for the $x$-axis, $y$-axis, origin and the lines $y= \pm x$. Then, noting that $L$ intersects the positive $x$-axis and the positive $y$-axis, we know that every orbit of (4) is a simple closed curve which is symmetric for the $x$-axis, $y$-axis, origin and the lines $y= \pm x$. Let $\left(x_{c}(t), y_{c}(t)\right)$ be the solution of (4) which satisfies $x_{c}(0)=c, y_{c}(0)=c \quad(c>0)$. Since the orbit of (4) is closed, the solution $\left(x_{c}(t), y_{c}(t)\right)$ is bounded. Because $g(x, y)$ satisfies the conditions (I) and (II), the solution $\left(x_{c}(t), y_{c}(t)\right)$ exists on $(-\infty,+\infty)[3]$.

Suppose that through the point $(c, c)$ the orbit of $(4)$ is $L_{c}$. Since $L_{c}$ is closed, the solution $\left(x_{c}(t), y_{c}(t)\right)$ is a periodic solution of (4). Let the period of $\left(x_{c}(t), y_{c}(t)\right)$ be $w(c)$. Because the solutions continuously depend on the 
initial conditions, it is easy to show that $w(c)$ is a continuous function. Noting that $L_{c}$ is symmetric for the $x$-axis, $y$-axis and

$$
\frac{d y}{d x}=-\frac{g(y, x)}{g(x, y)}<0 \quad(x>0, y>0),
$$

we have

$$
|x| \geq c \quad \text { or }|y| \geq c, \quad \forall(x, y) \in L_{c} .
$$

Then

$$
x^{2}+y^{2} \geq c^{2}, \quad \forall(x, y) \in L_{c}
$$

Let

$$
\begin{gathered}
m(c)=\inf \left\{x_{c}^{2}(t)+y_{c}^{2}(t), \quad 0 \leq t \leq w(c)\right\}, \\
\left.M(c)=\sup \left\{x_{c}^{2}(t)+y_{c}^{2}\right), \quad 0 \leq t \leq w(c)\right\} .
\end{gathered}
$$

Then $m(c) \geq 0 \quad M(c) \geq 0$. By (5), we obtain

$$
\lim _{c \rightarrow+\infty} m(c)=+\infty
$$

Since, under the conditions (I) and (II), the orbits of (4) are mutually disjoint [3], $M(c)$ is an increasing function and $\lim _{c \rightarrow 0^{+}} M(c)$ exists. Noting that $M(c) \geq 0$, we have $\lim _{c \rightarrow 0^{+}} M(c) \geq 0$. We claim that

$$
\lim _{c \rightarrow 0^{+}} M(c)=0 \text {. }
$$

Otherwise, we have $\lim _{c \rightarrow 0^{+}} M(c)=d>0$. Consider the orbit $L_{A}$ which passes through the point $A(\sqrt{d} / 2,0)$. Since $L_{A}$ is a simple closed curve which is symmetric for the lines $y= \pm x, L_{A}$ intersects the positive $y$-axis and the intersection point is $(0, \sqrt{d} / 2)$. Noting that $L_{A}$ is symmetric for the $x$-axis, the $y$-axis and $d y / d x>0$ on $x>0, y>0$, we have

$$
|x| \leq \sqrt{d} / 2, \quad|y| \leq \sqrt{d} / 2, \quad \forall(x, y) \in L_{A},
$$

and

$$
x^{2}+y^{2} \leq(\sqrt{d} / 2)^{2}+(\sqrt{d} / 2)^{2}=d / 2, \quad \forall(x, y) \in L_{A} .
$$

Let the intersection point of $L_{A}$ and $y=x$ be $(a, a)$. Then, we have $M(a) \leq d / 2$ which contradicts the fact that $\lim _{c \rightarrow 0^{+}} M(c)=d>0$ and establishes the claim that (7) holds.

Let $H(t)=\arctan y_{c}(t) / x_{c}(t)$. Then

$$
\begin{aligned}
2 \pi & =\int_{0}^{2 \pi} d H=\int_{0}^{w(c)} H^{\prime}(t) d t=\int_{0}^{w(c)} \frac{y_{c}^{\prime}(t) x_{c}(t)-x_{c}^{\prime}(t) y_{c}^{\prime}(t)}{x_{c}^{2}(t)+y_{c}^{2}(t)} d t \\
& =\int_{0}^{w(c)} \frac{x_{c}(t) g\left(y_{c}(t), x_{c}(t)\right)+y_{c}(t) g\left(x_{c}(t), y_{c}(t)\right)}{x_{c}^{2}(t)+y_{c}^{2}(t)} d t .
\end{aligned}
$$


Since (7) holds, $x_{c}^{2}(t)+y_{c}^{2}(t)$ uniformly tends to zero as $c \rightarrow 0^{+}$. Then, by (7), (8) and the conditions (c), (d), we have

$$
2 \pi=p \lim _{c \rightarrow 0^{+}} w(c) .
$$

Similarly, by (6), (8) and the conditions (c), (d), we have

$$
2 \pi=q \lim _{c \rightarrow+\infty} w(c) \text {. }
$$

Noting that $p<2 \pi / T<q$ or $q<2 \pi / T>p$ and (9), (10) hold, we obtain

$$
\lim _{c \rightarrow 0^{+}} w(c)>T, \quad \lim _{c \rightarrow+\infty} w(c)<T
$$

or

$$
\lim _{c \rightarrow 0^{+}} w(c)<T, \quad \lim _{c \rightarrow+\infty} w(c)>T .
$$

Hence, there exists $c^{*} \in(0,+\infty)$ such that $w\left(c^{*}\right)=T$ and the solution $\left(x^{*}(t), y^{*}(t)\right)$ which satisfies the initial conditions $x^{*}(0)=c^{*}, y^{*}(0)=c^{*}$ is a nonconstant periodic solution with period of $T$. The proof of the lemma is now complete.

THEOREM 1. Suppose that there is a function $g(x, y)$ such that

$$
f(x, y)=g(x, y)-c g(y, x)
$$

where $g(x, y)$ satisfies the conditions (I), (II). If the conditions (a), (b), (c) of the lemma and

$p<\frac{(1+4 n) \pi}{2 \tau}<q \quad$ or $\quad<\frac{(1+4 n) \pi}{2 \tau}<p \quad(n=m, n+1, \ldots, m+k-1)$

hold, then (1) has at least $k$ nonconstant oscillatory solutions, where $m$ is some nonnegative integer and $k$ is some positive integer.

Proof. By the lemma, (4) has a periodic solution with period of $T_{n}=$ $4 \tau /(1+4 m)$. Since $n=m, m+1, \ldots, m+k-1$, we obtain $k$ nonconstant solutions $\left(x_{n}(t), y_{n}(t)\right)$ of $(4)$, where $\left(x_{n}(t), y_{n}(t)\right)$ satisfies the initial conditions $x_{n}(0)=c_{n}, y_{n}(0)=c_{n}$ and the period of $\left(x_{n}(t), y_{n}(t)\right)$ is $w\left(c_{n}\right)=4 \tau /(1+4 n)$. Assume that, through the point $\left(c_{n}, c_{n}\right)$, the orbit of (4) is $L_{n}$. By the proof of the lemma, we know that $L_{n}$ is a simple closed curve which is symmetric for the $x$-axis, $y$-axis, origin and the lines $y= \pm x$. Since $L_{n}$ is symmetric for the origin, it is easy to show that the point $\left(-x_{n}(t),-y_{n}(t)\right) \in L_{n}$ for any $t \in(-\infty,+\infty)$ and that $\left(-x_{n}(t),-y_{n}(t)\right)$ is a solution of (4). Then the solution $\left(x_{n}(t), y_{n}(t)\right)$ will 
meet the solution $\left(-x_{n}(t),-y_{n}(t)\right)$ after a translation of time $\tau_{1}$, i.e. there is some $\tau_{1} \in\left(0, \frac{4 \tau}{1+4 n}\right)$ such that

$$
\begin{aligned}
& x_{n}(t)=-x_{n}\left(t+\tau_{1}\right)=x_{n}\left(t+2 \tau_{1}\right), \\
& y_{n}(t)=-y_{n}\left(t+\tau_{1}\right)=y_{n}\left(t+2 \tau_{1}\right) .
\end{aligned}
$$

Noting that $x_{n}(t)$ has period of $4 \tau /(1+4 n)$, by (12), we have

$$
2 \tau_{1}=h_{1} \cdot \frac{4 \tau}{1+4 n} \text { or } \tau_{1}=h_{1} \cdot \frac{2 \tau}{1+4 n}
$$

where $h_{1}$ is some positive integer. By $\tau_{1} \in\left(0, \frac{4 \tau}{1+4 n}\right)$ and $\tau_{1}=h_{1} \cdot \frac{2 \tau}{1+4 n}\left(h_{1}\right.$ is positive integer), we have $h_{1}=1$ and $\tau_{1}=2 \tau /(2+4 n)$. Then, by (12), we have

$$
\begin{aligned}
& x_{n}(t)=-x_{n}\left(t+\frac{2 \tau}{1+4 n}\right)=-x_{n}\left(t-\frac{2 \tau}{1+4 n}\right), \\
& y_{n}(t)=-y_{n}\left(t+\frac{2 \tau}{1+4 n}\right)=-y_{n}\left(t-\frac{2 \tau}{1+4 n}\right) .
\end{aligned}
$$

On the other hand, since the closed curve $L_{n}$ is symmetric for the $x$-axis, $y$-axis and the lines $y= \pm x$, it is easy to show that the point $\left(-y_{n}(t), x_{n}(t)\right) \in$ $L_{n}(t \in(-\infty,+\infty))$ and $\left(-y_{n}(t), x_{n}(t)\right)$ is a solution of (4). Then the solution $\left(x_{n}(t), y_{n}(t)\right)$ will meet the solution $\left(-y_{n}(t), x_{n}(t)\right)$ after a translation of time $\tau_{2}$, i.e. there is some $\tau_{2} \in\left(0, \frac{4 \tau}{1+4 n}\right)$ such that

$$
-y_{n}(t)=x_{n}\left(t+\tau_{2}\right), \quad x_{n}(t)=y_{n}\left(t+\tau_{2}\right) \text {. }
$$

By (14), we have

$$
x_{n}(t)=y_{n}\left(t+\tau_{2}\right)=-x_{n}\left(t+2 \tau_{2}\right) .
$$

By (13) and (15), we have

$$
x_{n}\left(t-\frac{2 \tau}{1+4 n}\right)=x_{n}\left(t+2 \tau_{2}\right)
$$

Then

$$
2 \tau_{2}+\frac{2 \tau}{1+4 n}=h_{2} \cdot \frac{4 \tau}{1+4 n} \quad \text { or } \quad \tau_{2}=\frac{4 \tau}{1+4 n} \cdot\left(\frac{h_{2}}{2}-\frac{1}{4}\right)
$$

where $h_{2}$ is some positive integer. By $\tau_{2} \in\left(0, \frac{4 \tau}{1+4 n}\right)$ and $(16)$, it is easy to see $h_{2}=1$ or 2 . Then $\tau_{2}=\frac{\tau}{1+4 n}$ or $\tau_{2}=\frac{3 \tau}{1+4 n}$. We choose $t_{0}$ such that the point $\left(x_{n}\left(t_{0}\right), y_{n}\left(t_{0}\right)\right)$ belongs to the first quadrant. Then $x_{n}\left(t_{0}\right)>0$, $y_{n}\left(t_{0}\right)>0$. Hence the point $\left(-y_{n}\left(t_{0}\right), x_{n}\left(t_{0}\right)\right)$ should belong to the second quadrant and the point $\left(-x_{n}\left(t_{0}\right),-y_{n}\left(t_{0}\right)\right)$ should belong to the third quadrant. 
By (13), and (14), we have

$$
\begin{gathered}
\left(x_{n}\left(t_{0}+\frac{2 \tau}{1+4 n}\right), y_{n}\left(t_{0}+\frac{2 \tau}{1+4 n}\right)\right)=\left(x_{n}\left(t_{0}\right),-y_{n}\left(t_{0}\right)\right), \\
\left(x_{n}\left(t_{0}+\tau_{2}\right), y_{n}\left(t_{0}+\tau_{2}\right)\right)=\left(-y_{n}\left(t_{0}\right), x_{n}\left(t_{0}\right)\right) .
\end{gathered}
$$

Then $\left(x_{n}\left(t_{0}+\tau_{2}\right), y_{n}\left(t_{0}+\tau_{2}\right)\right)$ belongs to the second quadrant and $\left(x_{n}\left(t_{0}+\frac{2 \tau}{1+4 n}\right), y_{n}\left(t_{0}+\frac{2 \tau}{1+4 n}\right)\right)$ belongs to the third quadrant. Hence $\tau_{2} \neq$ $\frac{3 \tau}{1+4 n}\left(h_{2} \neq 2\right)$ and $\tau_{2}=\frac{\tau}{1+4 n}\left(h_{2}=1\right)$.

By (14) and $\tau_{2}=\frac{\tau}{1+4 n}$, we obtain

$$
\begin{aligned}
x_{n}(t) & =y_{n}\left(t+\tau_{2}\right)=-x_{n}\left(t+2 \tau_{2}\right)=-y_{n}\left(t+3 \tau_{2}\right)=x_{n}(t+4 \tau) \\
& =y_{n}\left(t+5 \tau_{2}\right)=\cdots=y_{n}\left(t+(1+4 n) \tau_{2}\right)=y_{n}(t+\tau) .
\end{aligned}
$$

Then

$$
x_{n}(t-\tau)=y_{n}(t) .
$$

By (4) and (17), we have

$$
\begin{gathered}
\frac{d x_{n}(t)}{d t}=-g\left(x_{n}(t), y_{n}(t)\right)=-g\left(x_{n}(t), x_{n}(t-\tau)\right), \\
\frac{d x_{n}(t-\tau)}{d t}=\frac{d y_{n}(t)}{d t}=g\left(y_{n}(t), x_{n}(t)\right)=g\left(x_{n}(t-\tau), x_{n}(t)\right) .
\end{gathered}
$$

By (18), (19) and (11), we have

$$
\begin{aligned}
\frac{d\left(x_{n}(t)+c x_{n}(t-\tau)\right)}{d t} & =-g\left(x_{n}(t), x_{n}(t-\tau)\right)+c g\left(x_{n}(t-\tau), x_{n}(t)\right) \\
& =-f\left(x_{n}(t), x_{n}(t-\tau)\right) .
\end{aligned}
$$

Hence $x_{n}(t)$ is a periodic solution of (1). By the proof of the lemma, $x_{n}(t)$ is nonconstant oscillatory and has period of $\frac{4 \tau}{1+4 n}$. Since $n=m, m+$ $1, \ldots, m+k-1$ we obtain $k$ nonconstant oscillatory periodic solutions. The proof of Theorem 1 is now complete.

REMARK 1. By Theorem 1, if $p<+\infty, q=+\infty$ or $p=+\infty, q<+\infty$, then (1) has an infinite number of periodic solutions.

In the case $c=0, f(x, y)=F(y)$, we can choose $g(x, y)=F(y)$. Then, by Theorem 1, we have the following corollary:

\section{Corollary 1. Suppose that}

(a) $F(y)$ is a continuous odd function, $y F(y)>0(y \neq 0)$ and $\int^{+\infty} F(y) d y=+\infty$;

(b) $\lim _{y \rightarrow 0} F(y) / y=p, \lim _{y \rightarrow+\infty} F(y) / y=q$ and

$$
p<\frac{(1+4 n) \pi}{2 \tau}<q \quad \text { or } \quad q<\frac{(1+4 n) \pi}{2 \tau}<p,
$$


where $n=m, m+1, \ldots, m+k-1$ and $m$ is some nonnegative integer, $k$ is some positive integer. Then the equation

$$
x^{\prime}(t)=-F(x(t-\tau)) \quad(\tau>0)
$$

has at least $k$ nonconstant oscillatory periodic solutions. Specifically, if $p<$ $+\infty, q=+\infty$ or $p=+\infty, q<+\infty$, then (20) has an infinite number of periodic solutions.

REMARK 2. Corollary 1 generalises the result of Kaplan and Yorke [5].

\section{Some examples}

EXAMPLe 1. Consider

$$
(x(t)+x(t-\tau))^{\prime}=-a\left(x^{s}(t-\tau)-x^{s}(t)\right)
$$

where $a>0, \tau>0, s>1$ and $s$ is a ratio of two positive odd numbers. Then $c=1, f(x, y)=a\left(y^{s}-x^{s}\right)$. We choose $g(x, y)=a y^{s}$. It is easy to show that $g(x, y)$ satisfies the conditions of Theorem 1 and $p=0, q=$ $+\infty$. By Theorem 1 and Remark $1,(21)$ has an infinite number of periodic solutions.

EXample 2. Consider

$$
(x(t)-x(t-\tau))^{\prime}=-(x(t)+x(t-\tau)) \exp \left(-x^{2}(t)-x^{2}(t-\tau)\right),
$$

where $\tau>\frac{(1+4(k-1)) \pi}{2}$ and $k$ is some positive integer. Then $c=-1$ and $f(x, y)=(x+y) \exp \left(-x^{2}-y^{2}\right)$. We choose $g(x, y)=y \exp \left(-x^{2}-y^{2}\right)$. Hence, we have

$$
\frac{g(y, x)}{g(x, y)}=\frac{x \exp \left(-x^{2}-y^{2}\right)}{y \exp \left(-x^{2}-y^{2}\right)}=x \cdot \frac{1}{y} \quad(x \geq 0, y \geq b>0)
$$

and

$$
\frac{x g(y, x)+y g(x, y)}{x^{2}+y^{2}}=\exp \left(-x^{2}-y^{2}\right) .
$$

It is easy to show that $g(x, y)$ satisfies the conditions of Theorem 1 and

$$
q=0<\frac{(1+4 n) \pi}{2 \tau}<1=p, \quad n=0,1, \ldots, k-1 .
$$

Then, by Theorem $1,(22)$ has at least $k$ periodic solutions.

EXample 3. Consider

$$
(x(t)+c x(t-\tau))^{\prime}=-a\left(1+x^{2}(t)+x^{2}(t-\tau)\right)(x(t-\tau)-c x(t))
$$


where $a>0, \tau>0$ and $c$ is a constant. Then, $f(x, y)=a\left(1+x^{2}+y^{2}\right)(y-c x)$. We choose $g(x, y)=a\left(1+x^{2}+y^{2}\right) y$. Then we have

$$
\frac{g(y, x)}{g(x, y)}=\frac{a\left(1+y^{2}+x^{2}\right) x}{a\left(1+x^{2}+y^{2}\right) y}=x \cdot \frac{1}{y} \quad(x \geq 0, y \geq b>0)
$$

and

$$
\frac{x g(y, x)+y g(x, y)}{x^{2}+y^{2}}=a\left(1+x^{2}+y^{2}\right) .
$$

It is easy to show that $g(x, y)$ satisfies the conditions of Theorem 1 and $p=a, q=+\infty$. By Theorem 1 and Remark 1, (23) has an infinite number of periodic solutions. Indeed, it is easy to show that

$$
x_{n}(t)=\left(\frac{(1+4 n) \pi}{2 a \tau}-1\right)^{1 / 2} \cdot \sin \frac{(1+4 n) \pi t}{2 \tau}, \quad n=m, m+1, \ldots,
$$

are the periodic solutions of $(23)$, where $m=\left[\frac{2 \tau a-\pi}{4 \pi}\right]+1$. This is the same conclusion as we obtain by Theorem 1 and Remark 1 .

\section{References}

[1] R. K. Brayton, "Nonlinear oscillations in a distributed network", Quart. Appl. Math. 24 (1967) 289-301.

[2] R. K. Brayton and R. A. Willoughby, "On the numerical integration of a symmetric system of difference-differential equations of neutral type”, J. Math. Anal. Appl. 18 (1967) 182-189.

[3] J. K. Hale, Ordinary differential equations, (Wiley-Interscience, London, 1969).

[4] J. K. Hale, Theory of functional differential equations, (Springer Verlag, New York, 1977).

[5] J. L. Kaplan and J. A. Yorke, "Ordinary differential equations which yield periodic solutions of differential delay equations", J. Math. Anal. Appl. 48 (1974) 317-324.

[6] L. Z. Wen, "The existence of periodic solutions of a class of differential difference equations", Kexue Tongbao 32 (1987) 934-935. 